\title{
In vitro application of Ceratonia siliqua improved sperm parameters and chromatin quality after vitrifacation in normozoospermic aged men
}

\author{
Azita Faramarzi(D, Farank Aghaz(D, Mitra Bakhtiari(D) and Mozafar Khazaei* (D)
}

\begin{abstract}
Background: Vitrification is the main technique in the assisted reproductive technique (ART) labs. Sperm vitrification exposes sperm to damage. The aim of the present study was to evaluate the in vitro effect of Ceratonia siliqua (C. siliqua) application on sperm parameters and chromatin quality in normozoospermic aged men. Semen samples $(n=40)$ were collected from normozoospermic men over 45 years old. Each specimen was divided into four aliquots to form the subsequent groups: fresh (group I), vitrification without treatment (group II), vitrification with the medium supplemented by $20 \mathrm{\mu g} / \mathrm{ml} \mathrm{C}$. siliqua (group III), and vitrification with the thawing medium supplemented by $20 \mathrm{\mu g} / \mathrm{ml} \mathrm{C}$. siliqua (group IV). Sperm progressive motility, normal morphology and viability were assessed. Also, sperm chromatin quality was evaluated by aniline blue (AB), toluidine blue (TB), and sperm chromatin dispersion (SCD) staining.

Results: Vitrification caused a significant decrease in sperm progressive motility, normal morphology and viability as well as chromatin quality compared to fresh samples $(p<0.05)$. Supplementation of vitrification/thawing medium with C. siliqua significantly improved sperm progressive motility, normal morphology, viability, and chromatin quality compared to vitrification without any supplementation $(p<0.05)$.

Conclusions: The study showed that $C$. siliqua can improve the detrimental effect of vitrification on sperm parameters and chromatin quality of normozoospermic aged men.
\end{abstract}

Keywords: Human sperm, Vitrification, Ceratonia siliqua, Chromatin quality, Sperm parameters

\section{Background}

Infertility is an important problem in the twenty-first century. Male infertility is approximately estimated to be the cause of $50 \%$ of all infertility cases [1]. It is a concern since advanced paternal age may be related to increased abortion rates and different side effects on the newborn. Also, increased age might be related to reduced sperm quality [2]. However, it was recently reported that the average paternal age is increasing, and multiple cultural and socioeconomic factors affect fatherhood delay [3].

Human sperm cryopreservation has been industrialized, turning to the main issue in assisted reproductive

\footnotetext{
* Correspondence: mkhazaei1345@yahoo.com

Fertility and Infertility Research Center, Health Technology Institute, Kermanshah University of Medical Sciences, Kermanshah, Iran
}

technique (ART). Sperm cryopreservation has often been used for preservation of fertility ability before factors such as cytotoxic radiotherapy and chemotherapy, cytotoxic occupational exposure, certain diseases, and surgeries could lead ejaculatory and testicular failures [4, 5]. Finally, it is widely used to avoid second percutaneous epididymal sperm aspiration or testicular sperm extraction [6].

Vitrification is the most innovative method of sperm cryopreservation. It is based on the ultra-rapid decrease and increase in temperature by liquid nitrogen immersion. This technique is a safe, simple and inexpensive procedure [7].

In spite of the application of sperm vitrification in clinics, it has a limited success rate. It is well known that 
vitrification can cause damage to mitochondria, membrane, and genetic integrity, as well as the status of cell function and metabolism [8, 9]. Vitrification needs sperm preparation that removes the antioxidant source of seminal plasma [10]. In addition, it entails lipid peroxidation and increases in reactive oxygen species (ROS) levels in the sperm cytoplasm. Also, sperm freezing and warming might increase superoxide radical's generation, which induces DNA damage. Sperm DNA disintegrity brings low fertilization [11].

In order to improve sperm cryopreservation outcome, many factors such as the composition of freezing medium, the method of sample packaging and freezing/ thawing duration have been evaluated [12-14]. Freezing and thawing media supplementation with antioxidants agents are an effective way of overcoming the challenges of sperm vitrification [15]. Lately, numerous studies have evaluated herbal extract antioxidants application. Different herbal medicines have significant antioxidant effects [16-18].

Ceratonia siliqua (C. siliqua) has natural powerful antioxidant properties. It has several vitamins such as D, C, $\mathrm{E}$, folic acid, and niacin, many minerals such as sodium, potassium, phosphorus, calcium, and iron as well as polyphenol [17]. Phenolic components cause improvement of oxidative stress (OS) condition, have low side effects, and are inexpensive and accessible [19]. Also, the use of herbal extract may be an approach to know new medicine candidates.

To the best of our knowledge, there are no publications on the effects of $\mathrm{C}$. siliqua on cryopreservation in aged men. Although, we showed in our previous research that it has protective effects on sperm cryopreservation in young normozoospermic men [20]. Therefore, in the present study, we assessed the effect of in vitro application of C. siliqua on sperm parameters and chromatin quality after vitrification in aged normozoospermic patients.

\section{Methods}

\section{Study design}

This study was approved by the Ethical Committee of Kermanshah University of Medical Sciences (IR.KUMS.REC.1397.259), and the written informed consent was obtained from every patient participating in the research. Normozospermic semen samples $(n=40)$ were obtained from healthy men, aged 45-60 years, that were referred to the IVF Unit of Motazedi Hospital, Kermanshah, Iran. Sperm samples were collected by masturbation after 3-5 days of abstinence into sterile containers. After liquefaction at $37{ }^{\circ} \mathrm{C}, 10 \mu \mathrm{l}$ was removed from each sample and was analyzed [21]. In this study, patients with a history of varicocele, systemic diseases, accessory gland inflammation, smoking, alcohol intake, and late hormonal treatment were excluded.

Specimens were categorized normally according to the World Health Organization (WHO) guidelines. The seminal specimens were prepared using direct swim-up [21]. Every sample was divided into four aliquots to form subsequent groups: fresh (group I), cryopreserved without treatment (group II), cryopreserved + vitrification medium supplementation with $20 \mu \mathrm{g} / \mathrm{ml} \mathrm{C}$. siliqua (group III), cryopreserved without treatment but thawing medium supplementation with $20 \mu \mathrm{g} / \mathrm{ml} \mathrm{C}$. siliqua for 30 min incubation (group IV). This dose was according to previous research [20]. All assessments were done before cryopreservation and after thawing.

\section{Sperm parameters analysis}

Sperm motility and normal morphology were assessed for 200 spermatozoa in each sample. Motility was evaluated by the Makler chamber and light microscopy (Olympus, Tokyo, Japan). Sperm motility was categorized into (\%) progressive, non-progressive, and immotile. Eosin-nigrosin staining and Papanicolaou staining were used for viability and morphology assessment, respectively [21].

\section{Extract preparation}

Experts of Pharmaceutical Sciences Research Center, Kermanshah University of Medical Sciences, confirmed C. siliqua. After cleaning and drying, $10 \mathrm{~g}$ of leaf and fruit powder was dissolved in $1000 \mathrm{cc}$ distilled water. Then, the solution was filtered by paper (Whatman No. $2 \mathrm{UK})$. It was centrifuged at $5000 \mathrm{~g}$ for $20 \mathrm{~min}$, and then, the pellet was put in a proper glass dish at $40{ }^{\circ} \mathrm{C}$ bathwater. After water vaporization, it was added to $10 \mathrm{cc}$ Ham's-F10 and stored at $4{ }^{\circ} \mathrm{C}$.

\section{Chromatin quality evaluation}

Aniline Blue (AB) and Toluidine Blue (TB) staining as well as chromatin dispersion (SCD) test were used for chromatin assessment.

\section{$A B$ staining}

Each sperm smear was air-dried and fixed in 3\% buffered glutaraldehyde in $0.2 \mathrm{M}$ phosphate buffer ( $\mathrm{pH}$ 7.2) for $30 \mathrm{~min}$ at room temperature. The slides were placed with $5 \%$ aqueous $\mathrm{AB}$ stain in $4 \%$ acetic acid ( $\mathrm{pH} 3.5$ ) for $5 \mathrm{~min}$. At least 200 spermatozoa were counted in each smear using light microscopy (Olympus Co., Tokyo, Japan). Pale blue/colorless stained was reported as normal (AB-). Dark blue-stained was considered as abnormal sperm $(\mathrm{AB}+)$ [22]. 


\section{TB staining}

The smears were air-dried and fixed in fresh ethanol (96\%) acetone $(1: 1)$ at $4{ }^{\circ} \mathrm{C}$ for $30 \mathrm{~min}$. Then, they were hydrolyzed with $0.1 \mathrm{~N}$ hydrochloric acids at $4{ }^{\circ} \mathrm{C}$ for 5 min, and the smears were washed three times in distilled water for $2 \mathrm{~min}$. After that, the slides were stained with $0.05 \%$ TB in 50\% Mcllvain's citrate phosphate Buffer $(\mathrm{pH} 3.5)$ for $10 \mathrm{~min}$ at room temperature. For each slide, 200 spermatozoa were examined by light microscopy (Olympus Co., Tokyo, Japan). Sperms indicating light blue were considered as normal (TB-), and violet/dark blue was reported as abnormal (TB+) [23].

\section{SCD test}

A sperm aliquot of each specimen $(50 \mu \mathrm{l})$ was mixed with a small melting point agarose. It was pipetted on a glass slide and covered by a coverslip. The slides were put on a surface at a temperature of $2{ }^{\circ} \mathrm{C}$ to $8{ }^{\circ} \mathrm{C}$ for 5 min to solidify. They were immersed in lysis and denaturation solutions. The slides were washed in distilled water and then, dehydrated in $70 \%, 90 \%$, and $100 \%$ ethanol for $2 \mathrm{~min}$ at room temperature and air-dried. The stained slides were washed in distilled water. After drying, at least 200 spermatozoa were examined by light microscopy (Olympus Co., Tokyo, Japan). Large/ medium-sized halo head was considered as intact chromatin, and small/without halo head was reported as damaged chromatin [24].

\section{Sperm vitrification and thawing}

The modified straw to straw method was done for vitrification (close system) and thawing according to Isachenko et al. [25]. Sperm suspension was mixed with Ham's F10 medium supplemented with 5\% HSA/0.5 $\mathrm{mol} / \mathrm{l}$ sucrose (1:1). One hundred microliters of this solution was placed on a $0.25-\mathrm{ml}$ plastic sterile straw, and then it was put into a $0.5-\mathrm{ml}$ straw. Finally, the straw end was sealed. All procedures were performed horizontally and carried out at room temperature. The $0.5 \mathrm{ml}$ straws were directly plunged into liquid nitrogen and stored for 10 days.

For thawing, one end of the straws was cut and the vitrified sperm was added to pre-warmed Ham's F10 with 5\% HAS in a conical tube. The simple wash, sperm analysis, and chromatin assessment were done immediately as performed before vitrification.

\section{Statistical analysis}

Statistical analyses were done by the Statistical Package for the Social Sciences 20 (SPSS Inc., Chicago, IL). The distribution of the results was analyzed using the Kolmogorov-Smirnov test. The data were shown as mean \pm $\mathrm{SD}$. The parametric data were examined by one-way ANOVA test and the Tukey post-test. $p<0.05$ was considered significant.

\section{Results}

The percentage of the sperm progressive motility, normal morphology, and viability significantly decreased in all vitrified groups compared to fresh groups $(p<0.05)$. However, vitrification and thawing media supplementation with C. siliqua significantly increased these parameters compared to vitrified groups without any supplementation $(p<0.05)$, Table 1.

Table 2 demonstrates the data on chromatin condensation. $\mathrm{AB}, \mathrm{TB}$, and SDF considerably increased in vitrified groups $(p<0.05)$. But in C. siliqua groups, the rates of chromatin condensation damage were statistically decreased $(p<0.05)$.

\section{Discussion}

This study showed that progressive motility, normal morphology, viability, and chromatin condensation significantly decreased following sperm vitrification. But, C. siliqua recovered detrimental effects of vitrification on sperm parameters and in men $<45$ years. Also, C. siliqua improved sperm chromatin condensation (reduced $A B$, $\mathrm{TB}$, and $\mathrm{SCD}$ ) in these men.

Sperm vitrification procedure is a beneficial method while it poses a real challenge in ART centers. Sperm motility, viability, morphology, and chromatin integrity can be negatively affected by vitrification. Therefore, it may have detrimental effects on ART outcomes [26]. Moreover, there has been a trend to postpone fatherhood to later age over the past decade. Advanced paternal age might be accompanied by poor sperm quality

Table 1 Effect of vitrification and vitrification/thawing media supplementation with C siliqua on sperm progressive motility, normal morphology, and viability

\begin{tabular}{lllll}
\hline Variables & Group I & Group II & Group III & Group IV \\
\hline Progressive motility (\%) & $41.15 \pm 7.14$ & $25.19 \pm 2.01^{*}$ & $32.02 \pm 3.24^{*} \#$ & $30.31 \pm 2.01^{*} \#$ \\
Normal morphology (\%) & $8.18 \pm 1.2$ & $5.14 \pm 1.12^{*}$ & $6.12 \pm 1.12^{*} \#$ & $7.81 \pm 3.21^{*} \#$ \\
Viability (\%) & $66.40 \pm 3.1$ & $44.19 \pm 2.01^{*}$ & $57.12 \pm 1.12^{*} \#$ & $55.12 \pm 2.36^{*} \#$ \\
\hline
\end{tabular}

Group I fresh sperm, Group II vitrified sperm, Group III C. siliqua added to vitrification medium, Group IV C. siliqua added to thawing medium. The level of statistical significance was set at $p<0.05$ and was shown with $^{*}$ and \#

*Difference between group I and other groups

\#Difference between group II and groups III and IV 
Table 2 Effect of vitrification and vitrification/thawing media supplementation with C. siliqua on sperm AB, TB, and SCD

\begin{tabular}{lllll}
\hline Variables & Group I & Group II & Group III & Group IV \\
\hline AB (+) (\%) & $17.60 \pm 3.21$ & $31.21 \pm 4.11^{*}$ & $19.21 \pm 2.26^{*} \#$ & $19.34 \pm 3.21{ }^{*} \#$ \\
TB (+) (\%) & $28.36 \pm 2.36$ & $48.12 \pm 1.19^{*}$ & $37.12 \pm 2.36^{*} \#$ & $38.19 \pm 1.93{ }^{*} \#$ \\
SCD (+) (\%) & $15.47 \pm 1.63$ & $27.67 \pm 2.14^{*}$ & $18.26 \pm 2.9$ * \# $^{*}$ & $19.01 \pm 1.12{ }^{*} \#$
\end{tabular}

Group I fresh sperm, Group /I vitrified sperm, Group III C. siliqua added to vitrification medium, Group IV C. siliqua added to thawing medium. The level of statistical significance was set at $p<0.05$ and was shown with ${ }^{*}$ and \#

*Difference between group I and other groups

\#Difference between group II and groups III and IV

and as a result poor ART outcome. An important issue is that advanced aged men having their first baby often partnered with older women [2]. Thus, various studies on the improvement of ART outcomes in aged men are essential.

Because of the small amount of cytoplasm in sperm, which is removed during spermiogenesis, seminal plasma has an important role in ROS defense through several nonenzymatic and enzymatic antioxidants. Nonetheless, extender dilution before vitrification decreases the antioxidants constituents so that the resulting sperm is vulnerable to OS [27]. The proper level of ROS is necessary for sperm function. It is well known that a high level of ROS has an important role in cryopreservation pathology. ROS has detrimental effects on sperm chromatin, proteins, and lipids after cryopreservation. Extra and intracytoplasmic physiological scavenger systems can improve the detrimental effects of ROS [28]. Therefore, adding antioxidant agents to the vitrification/thawing medium might be an effective approach to minimize spermatozoa cryoinjury. Several antioxidants have been utilized for improving the sperm cryopreservation technique. Despite hopeful data, their protective role during freezing is limited and partial [26].

Several herbal medicines have been investigated for their sperm quality improvement properties, especially in Iran $[29,30]$. In addition, some studies revealed that C. siliqua has protective effects on gastrointestinal injuries induced by alcohol intake [31], antibacterial feature [32], anticancer feature [33], anti-diabetic activity [34], and antioxidant property [35]. It has been shown that C. siliqua improved the detrimental effects of free radicals of liver induced by alcohol [36].

In accordance with our study, C. siliqua improved sperm parameters such as concentration, viability, and normal morphology of sperm. Also, it increased superoxide dismutase enzyme, catalase and thiol levels, and germinal epithelium thickness, as well as decreased malondialdehyde level in busulfan administrated mice [17]. Also, we studied different doses of vitrification and thawing media supplementation by $C$. siliqua on sperm quality in young men. Our previous work showed that $20 \mu \mathrm{g} / \mathrm{ml} \mathrm{C}$. siliqua improved sperm quality in these men [20].
However, the exact mechanism of the protective effect of C. siliqua on sperm cryopreservation is not known. High-performance liquid chromatography (HPLC) analysis showed that $\mathrm{C}$. siliqua had flavonoids such as catechin gallate, epicatechin, quercetin glycosides, polyphenols of gallic acid, ellagic acid epigallocatechin gallate, and anthocyanins such as proanthocyanidins and ellagitannins [37]. Antioxidant activity is the main property of these compounds [38]. This property of C. siliqua may bring improvement of cryopreservation damage.

It is important to note that the method of extract preparation is not suitable for use in ICSI programs in its current form. The dried leaflet of the plant may have impurities and its safety as described may have deleterious effects on embryos. Therefore, it is suggested that this herbal extract be purified to pass quality control measures in order to ensure its purity. Limitations of the present study included a lack of information about oxidant and antioxidant measurement, as well as the use of the crude form of the herbal extract.

\section{Conclusion}

The study demonstrated that supplementation of vitrification/thawing medium with C. siliqua improved sperm parameters and chromatin condensation in normozoospermic aged men. It is suggested that this herbal extract be purified, and the resulting new effective antioxidant is discovered to improve sperm cryopreservation outcome in clinics.

\section{Abbreviations}

ART: Assisted reproductive technique; C. siliqua: Ceratonia siliqua; AB: Aniline blue; TB: Toluidine blue; SCD: Sperm chromatin dispersion; ROS: Reactive oxygen species

\section{Acknowledgments}

Our sincere appreciation goes to Motazedi Infertility Center.

\section{Authors' contributions}

AF designed the study and wrote the article draft. FA performed the sperm examination and cryopreservation. MB analyzed and interpreted the data. MK administered technical and logistic support. All authors have read and approved the manuscript and ensure that this is the case.

Funding

Kermanshah University of Medical Sciences

Availability of data and materials

The data will not be shared because it is the policy of our center. 


\section{Ethics approval and consent to participate}

This study was approved by the Ethical Committee of Kermanshah University of Medical Sciences (IR.KUMS.REC.1397.259), and written informed consent was obtained from every patient who participated in the research.

\section{Consent for publication}

Not applicable.

\section{Competing interests}

The authors declare that they have no competing interests.

Received: 11 September 2019 Accepted: 31 October 2019 Published online: 28 November 2019

\section{References}

1. Katz DJ, Teloken P, Shoshany O (2017) Male infertility-the other side of the equation. Aust Fam Physician. 46(9):641

2. Stone BA, Alex A, Werlin LB, Marrs RP (2013) Age thresholds for changes in semen parameters in men. Fertility and sterility. 100(4):952-958

3. Avellino G, Theva D, Oates RD (2017) Common urologic diseases in older men and their treatment: how they impact fertility. Fertility and sterility. 107(2):305-311

4. Di Santo M, Tarozzi N, Nadalini M, Borini A (2012) Human sperm cryopreservation: update on techniques, effect on DNA integrity, and implications for ART. Adv Urol. 2012

5. Hamada A, Wasik M, Gupta S, Agarwal A. Sperm banking: indications and regulations. Infertility: diagnosis, management and IVF Jaypee Brothers Medical Publishing, New Delhi. 2012:409-434.

6. Rarani FZ, Golshan-Iranpour F, Dashti GR. Correlation between sperm motility and sperm chromatin/DNA damage before and after cryopreservation and the effect of folic acid and nicotinic acid on post-thaw sperm quality in normozoospermic men. Cell and tissue banking. 2019:1-12.

7. Aizpurua J, Medrano L, Enciso M, Sarasa J, Romero A, Fernández MA et al (2017) New permeable cryoprotectant-free vitrification method for native human sperm. Human Reproduction. 32(10):2007-2015

8. Johnston S, Satake N, Zee Y, López-Fernández C, Holt WV, Gosalvez J (2012) Osmotic stress and cryoinjury of koala sperm: an integrative study of the plasma membrane, chromatin stability and mitochondrial function. Reproduction. 143(6):787-797

9. Kopeika J, Thornhill A, Khalaf Y (2014) The effect of cryopreservation on the genome of gametes and embryos: principles of cryobiology and critical appraisal of the evidence. Hum Reprod Update 21(2):209-227

10. Donnelly ET, McClure N, Lewis SE (2001) Cryopreservation of human semen and prepared sperm: effects on motility parameters and DNA integrity. Fertility and sterility. 76(5):892-900

11. Agarwal A, Prabakaran SA, Said TM (2005) Prevention of oxidative stress injury to sperm. Journal of Andrology. 26(6):654-660

12. Ansari MS, Rakha BA, Andrabi SM, Akhter S (2011) Effect of straw size and thawing time on quality of cryopreserved buffalo (Bubalus bubalis) semen. Reprod Biol. 11(1):49-54

13. Meamar M, Zribi N, Cambi M, Tamburrino L, Marchiani S, Filimberti E et al (2012) Sperm DNA fragmentation induced by cryopreservation: new insights and effect of a natural extract from Opuntia ficus-indica. Fertility and sterility. 98(2):326-333

14. Pavlovych O, Revenko O, Gapon G (2016) Optimization of thawing regimen for cryopreserved human sperm at normo-and pathospermia. Probl Cryobiology Cryomedicine. 26(1):45-52

15. Kalthur G, Raj S, Thiyagarajan A, Kumar S, Kumar P, Adiga SK (2011) Vitamin E supplementation in semen-freezing medium improves the motility and protects sperm from freeze-thaw-induced DNA damage. Fertility and Sterility. 95(3):1149-1151

16. Forouzanfar M, Abid A, Hosseini SM, Hajian M, Esfahani MHN (2013) Supplementation of sperm cryopreservation media with cell permeable superoxide dismutase mimetic agent (MnTE) improves goat blastocyst formation. Cryobiology. 67(3):394-397

17. Vafaei A, Mohammadi S, Fazel A, Soukhtanloo M, Pour AM, Beheshti F (2018) Effects of carob (Ceratonia siliqua) on sperm quality, testicular structure, testosterone level and oxidative stress in busulfan-induced infertile mice. Pharm Sci. 24(2):104-111
18. Khazaei M, Pazhouhi M, Khazaei S (2018) Evaluation of Hydro-Alcoholic Extract of Trifolium Pratens L. for Its Anti-Cancer Potential on U87MG Cell Line. Cell journal. 20(3):412-421

19. Amessis-Ouchemoukh N, Ouchemoukh S, Meziant N, Idiri Y, Hernanz D, Stinco CM et al (2017) Bioactive metabolites involved in the antioxidant, anticancer and anticalpain activities of Ficus carica L., Ceratonia siliqua L. and Quercus ilex L. extracts. Ind Crops Prod 95:6-17

20. Faramarzi A, Aghaz F, Jahromi MG, Bakhtiari M, Khazaei M (2019) Does supplementation of sperm freezing/thawing media with Ceratonia siliqua improve detrimental effect of cryopreservation on sperm parameters and chromatin quality in normozoospermic specimens? Cell Tissue Banking. 20(3):403-409

21. Organization $\mathrm{WH}$. WHO laboratory manual for the examination and processing of human semen. 2010.

22. Franken D, Franken C, De La Guerre H, De Villiers A (1999) Normal sperm morphology and chromatin packaging: comparison between aniline blue and chromomycin A3 staining. Andrologia. 31(6):361-366

23. Rosenborg L, Rao K, Björndahlt L, Kvist U, Pousette A, Ákerlof E et al (1990) Changes in human sperm chromatin stability during preparation for in-vitro fertilization. Int J Androl. 13(4):287-296

24. Viloria T, Garrido N, Fernández JL, Remohí J, Pellicer A, Meseguer M (2007) Sperm selection by swim-up in terms of deoxyribonucleic acid fragmentation as measured by the sperm chromatin dispersion test is altered in heavy smokers. Fertility and Sterility. 88(2):523-525

25. Isachenko V, Maettner R, Petrunkina A, Mallmann P, Rahimi G, Sterzik K et al (2011) Cryoprotectant-free vitrification of human spermatozoa in large (up to $0.5 \mathrm{~mL}$ ) volume: a novel technology. Clinical laboratory. 57(9-10):643-650

26. Liu J, Wang W, Liu X, Wang X, Wang J, Wang Y et al (2018) Supplementation of cryopreservation medium with TAT-Peroxiredoxin 2 fusion protein improves human sperm quality and function. Fertility and sterility. 110(6):1058-1066

27. Ben Abdallah F, Dammak I, Attia H, Hentati B, Ammar-Keskes L (2009) Lipid peroxidation and antioxidant enzyme activities in infertile men: correlation with semen parameter. J Clin Lab Anal. 23(2):99-104

28. Thomson LK, Fleming SD, Aitken RJ, De luliis GN, Zieschang J-A, Clark AM (2009) Cryopreservation-induced human sperm DNA damage is predominantly mediated by oxidative stress rather than apoptosis. Hum Reprod. 24(9):2061-2070

29. Nejatbakhsh F, Shirbeigi L, Rahimi R, Abolhassani H (2016) Review of local herbal compounds found in the Iranian traditional medicine known to optimise male fertility. Andrologia. 48(8):850-859

30. Beheshti B, Bandariyan E, Hemati M (2019) Effects of Hibiscus Sabdariffa aqueous extract on spermatogenesis and sperm parameters of mice. Herbal Med J 3(3)

31. Rtibi K, Jabri MA, Selmi S, Souli A, Sebai H, El-Benna J et al (2015) Gastroprotective effect of carob (Ceratonia siliqua L.) against ethanolinduced oxidative stress in rat. BMC Complement Altern Med 15(1):292

32. Meziani S, Oomah BD, Zaidi F, Simon-Levert A, Bertrand C, Zaidi-Yahiaoui R (2015) Antibacterial activity of carob (Ceratonia siliqua L.) extracts against phytopathogenic bacteria Pectobacterium atrosepticum. Microb Pathog. 78:95-102

33. Corsi L, Avallone R, Cosenza F, Farina F, Baraldi C, Baraldi M (2002) Antiproliferative effects of Ceratonia siliqua L. on mouse hepatocellular carcinoma cell line. Fitoterapia. 73(7-8):674-684

34. Rtibi K, Selmi S, Grami D, Saidani K, Sebai H, Amri M et al (2017) Ceratonia siliqua L.(immature carob bean) inhibits intestinal glucose absorption, improves glucose tolerance and protects against alloxan-induced diabetes in rat. J Sci Food Agric. 97(8):2664-2670

35. Custódio L, Escapa AL, Fernandes E, Fajardo A, Aligué R, Alberício F et al (2011) Phytochemical profile, antioxidant and cytotoxic activities of the carob tree (Ceratonia siliqua L.) germ flour extracts. Plant Foods Hum Nutr. 66(1):78-84

36. Souli A, Sebai H, Chehimi L, Rtibi K, Tounsi H, Boubaker S et al (2015) Hepatoprotective effect of carob against acute ethanol-induced oxidative stress in rat. Toxicol Ind Health. 31(9):802-810

37. Hadi MY, Hameed IH, Ibraheam IA (2017) Ceratonia siliqua: Characterization, Pharmaceutical Products and Analysis of Bioactive Compounds: A Review. Res J Pharm Technol. 10(10):3585-3589

38. Alzoubi KH, Alibbini S, Khabour OF, El-Elimat T, Al-zubi M, Alali FQ (2018) Carob (Ceratonia siliqua L.) Prevents Short-Term Memory Deficit Induced by Chronic Stress in Rats. J Mol Neurosci. 66(3):314-321

\section{Publisher's Note}

Springer Nature remains neutral with regard to jurisdictional claims in published maps and institutional affiliations. 